\title{
Polymorphic Variation in the Structure of Aerial Sclerotia of Coprinus cinereus
}

\author{
By F. V. HEREWARD AND DAVID MOORE \\ Department of Botany, The University, Manchester M13 9PL
}

(Received 7 November 1978)

\begin{abstract}
Aerial sclerotia of different wild-type strains of Coprinus cinereus differed in their internal structure. The more common of the two polymorphic forms (called the Z-type) had a rind which was only one cell thick; the other form (the H-type) had a rind many cells thick which extended to at least half the diameter of the sclerotium. Visible light microscopy, ultraviolet fluorescence microscopy and scanning electron microscopy were used to characterize the sclerotia formed by a range of dikaryotic mycelia to establish the genetic relationships between the polymorphic structures. The Z-type is the wild-type and genetically dominant form. The H-type is a naturally occurring variant caused by an allele of the gene scl-1 which we designate $s c l-1^{\mathrm{H}}$. The $s c l-1^{\mathrm{H}}$ allele is recessive to wild-type but is dominant to the previously known allele of scl-1 (now designated $\mathrm{scl}-1^{\circ}$ ). Strains carrying $\mathrm{scl}-1^{\circ}$ fail to produce sclerotia and since its allele $\mathrm{scl}-\mathrm{I}^{\mathrm{H}}$ causes the formation of sclerotia having an abnormal proliferation of cells, particularly in the rind, it is suggested that the scl-1 gene may normally be involved in the control of the disposition and extent of tissue layers during sclerotium development.
\end{abstract}

\section{INTRODUCTION}

The sclerotia of Coprinus cinereus are small, multicellular, globose structures which are probably perennating bodies able to survive adverse conditions. Both monokaryotic and dikaryotic mycelia are capable of forming them and they are a totally vegetative expression of morphogenesis. According to Kemp (1975), this is a feature unique to C. cinereus among the immediate relatives of this species. Dikaryotic mycelia are also capable of producing carpophores as part of the sexual cycle. Both sclerotia and carpophores develop from undifferentiated mycelia through an organized process of hyphal growth and branching which forms an aggregate in which cellular differentiation occurs. The early stages of this sequence are common to the processes of carpophore primordium and sclerotium formation (Matthews \& Niederpruem, 1972; Waters et al., 1975b), the cell mass becoming committed to one or other of these pathways as a result of the interaction of environmental factors like light (Morimoto \& Oda, 1973; Lu, 1974) and nutrition (Moore \& Jirjis, 1976) with the genetic factors controlling differentiation. This ability to respond to environmental conditions by the production of alternative structures provides an ideal experimental system for investigation of gene control during morphogenesis in eukaryotes. The present observations were made on strains of diverse origin as part of a study relating sclerotium structure to the genotype of the mycelium.

There is a disagreement in the literature about the internal structure of mature sclerotia. The first study (Volz \& Niederpruem, 1970) reported only partial success with electron microscopy and relied almost totally on light microscopy of wax-embedded sections; the study revealed that mature sclerotia possessed an outer unicellular rind layer, composed of 
cells with thickened and pigmented walls, which enclosed a medulla composed of a compact mass of thin-walled bulbous cells and accompanying hyphae. A later study (Waters et al., 1975a) made considerable use of transmission electron microscopy and though the cultures used were closely related to those employed by Volz \& Niederpruem (1970) the structure reported was very different; the sclerotia were found to have a multilayered rind enclosing a compact medulla composed predominantly of thick-walled cells (Waters et al., 1972, 1975a). Waters et al. (1975a) suggested that the differences in the reported structures could be accounted for by assuming that Volz \& Niederpruem (1970) had not distinguished aerial and submerged sclerotia. Sclerotia formed by the submerged mycelium and within the agar medium were shown to have a single-layered rind and very open internal structure (Waters et al., 1975a) and some of the photographs published by Volz \& Niederpruem (1970) certainly show sections of sclerotia enclosed within submerged mycelium, whilst others show sections of aerial structures. In the present work, however, the structures of sclerotia produced by strains of different origins have been scrutinized, and a genuine polymorphism in the structure of aerial sclerotia has been found. These polymorphic structures are compared and we explore some aspects of the genetic basis of the polymorphism.

\section{METHODS}

Organism. The correct name of the organism used is Coprinus cinereus (Schaeff. ex Fr.) S. F. Gray sensu Konr. (Pinto-Lopes \& Almeida, 1970) although in previous work it has been called C. lagopus. Most of the observations have been made using dikaryotic mycelia formed from the monokaryons numbered $\mathrm{B} 1, \mathrm{BC} 9 / 6,6$, $\mathrm{H} 1, \mathrm{H} 9,2 \mathrm{H} 1$ and $\mathrm{ZBw601} / 40,40$ which were used in earlier work on sclerotium structure and morphogenesis (Waters et al., $1975 a, b$ ).

Conditions of culture. Colonies were usually grown on maltose-CM medium (Stewart \& Moore, 1974), contained in $9 \mathrm{~cm}$ Petri dishes, and incubated in the dark at $37^{\circ} \mathrm{C}$.

Microscopic methods. Sclerotia were examined whole, fractured or sectioned, but all material was first fixed overnight at room temperature in $4 \%$ (v/v) glutaraldehyde buffered with 20 mM-phosphate buffer to pH 7. Subsequently, whole sclerotia were dehydrated in ethanol, dried in air from amyl acetate, sprinkled on to scanning electron microscopy stubs covered with double-sided adhesive tape and coated with gold.

Sections were cut from wax-embedded material. After fixation, the sclerotia were dehydrated through an ethanol series and concurrently impregnated with 2-methylpropan-2-ol (TBA) (Jensen, 1962); after three changes of pure TBA, the specimens were embedded in wax. Sections were cut and mounted on glass slides. Without removing the wax, they were stained with the fluorescent brighteners aniline blue, Photine LV or Tinopal BOPT and examined by ultraviolet light microscopy. After removing the wax, sections were stained with the periodic acid-Schiff reagent (PAS) to detect glycogen (Waters et al., 1975 b). De-waxed sections were also examined by scanning electron microscopy by cutting out the relevant piece of the glass microscope slide, mounting it on a stub and coating with gold. It was possible to apply all three procedures (ultraviolet fluorescence microscopy, PAS-staining and scanning electron microscopy) consecutively to the same sections, and this approach was used extensively to compare the polymorphic sclerotial structures. However, different sclerotium morphologies were readily distinguished after PAS-staining so this was used as a routine procedure for diagnosis of the type of sclerotium produced by a particular mycelium.

Genetic techniques. Sclerotia are produced by monokaryotic and dikaryotic mycelia. It was thus possible to establish the 'sclerotial phenotypes' of two monokaryons and then to investigate the phenotype of the sclerotia formed by their dikaryon. In this way, genetic variants were tested for dominance relationships and complementation patterns so that allelic relationships could be established. No attempts were made to test for segregations affecting sclerotium morphology.

\section{RESULTS AND DISCUSSION}

In previous work (Waters et al., 1975b), it was reported that wild-type strains differed in their ability to form aerial sclerotia; of 47 monokaryotic strains studied five were found to be incapable of forming sclerotia and subsequent genetic tests revealed that four distinct genes were involved. In this earlier study only the ability or inability to produce sclerotia was investigated; no attempt was made to compare the structures of the sclerotia 
formed, and only the structure of aerial sclerotia produced by the monokaryon $\mathrm{H} 1$ was described in detail.

Further investigations of the phenotype of aerial sclerotia produced by different isolates from the wild have shown that two distinct structures can be found. The structure which has previously been described as being produced by strain $\mathrm{H} 1$ will be called the $\mathrm{H}$-type. The second polymorphic form was first observed by us in sclerotia produced by the monokaryon with the code number ZBw601/40,40 and will be called the Z-type. The characteristic difference between the two types of sclerotia was that the Z-type had a rind which was only one cell thick (Figs 1, 2 and 4) and thus its structure is comparable to the morphology described by Volz \& Niederpruem (1970), whereas the H-type had a rind structure many cells thick (Figs 3, 5 and 6) as was described by Waters et al. (1975a). The internal structure of the two types was very similar: both contained scattered inflated cells, often with PAS-positive contents (Figs 1 and 3), within a compact cellular matrix (Figs 4 and 5). The internal structure bears a close resemblance to that described for sclerotia of Sclerotinia sclerotiorum and, as Colotelo (1974) observed in this species, the exact morphology of the medullary tissue depended on the stage of development of the specimen and also on the degree of hydration of the mucilaginous matrix. Fluorescent brighteners revealed the cellular structure within the medulla. This was often hyphal (Figs 2 and 6), even in the specimens in which visiblelight microscopy revealed a very amorphous structure (compare Figs 2 and 4 or 5 and 6). It seems probable, therefore, that the internal hyphal cells give rise to the amorphous matrix while still retaining their original shape. The 'thick secondary wall' described earlier (Waters et al., 1972) may represent a mucilage layer and the medullary structure is probably much less substantial than might be imagined from existing descriptions (Waters et al., 1972, 1975a).

The genetic basis of the polymorphism was investigated by constructing dikaryons from selected monokaryotic strains, the sclerotia produced being scored as H-type or Z-type by light microscopy of wax-embedded sections. The monokaryons used for dikaryon construction included $\mathrm{H1}$ and ZBw601/40,40 and a number of others known to be unable to produce sclerotia because of genetic defects. Three loci (scl-1, scl-2 and scl-3) at which alleles which prevent sclerotium formation have been identified were mapped genetically by Waters et al. $(1975 b)$ and representatives of these were combined in dikaryons together with $\mathrm{H} 1$ and ZBw601/40,40. Details of these dikaryons, together with the type of sclerotium produced, are shown in Table 1. Certain conclusions can be derived from these results.

1. The dikaryon made from the H-type producer $(\mathrm{H} 1)$ and the Z-type producer (ZBw601/ 40,40 ) formed Z-type sclerotia indicating that this is the dominant phenotype.

2. All dikaryons made between the Z-type producer (ZBw601/40,40) and sclerotiumnegative strains produced Z-type sclerotia showing that the inability to form sclerotia is recessive and suggesting that the Z-type morphology should be described as the 'wild-type'.

3. Dikaryons made between sclerotium-negative strains form Z-type sclerotia showing that these distinct genes complement one another in doubly-heterozygous dikaryons, which again supports the view that the Z-type morphology is the wild-type.

4. Dikaryons made between $\mathrm{H} 1$ and the two sclerotium-negative monokaryons B1 (scl-2) and $2 \mathrm{H} 1(\mathrm{scl}-3)$ produce Z-type sclerotia indicating that the $\mathrm{H}$-type phenotype of $\mathrm{H} 1$ is a sclerotium developmental defect which is genetically recessive and is complemented by dominant factors carried by the two sclerotium-negative strains.

5. Dikaryons made between $\mathrm{H} 1$ and either of the two related strains which carry the sclerotium-negative gene scl-1 ( $\mathrm{H} 9$ and $\mathrm{BC} 9 / 6,6)$ produce $\mathrm{H}$-type sclerotia suggesting that these sclerotium-negative strains do not carry genes able to complement the defect which controls the H-type morphology.

The most straightforward interpretation of these observations is that the H-type sclerotium structure is the phenotype resulting from a genetic defect which is an allele of the gene scl-1; we designate it $s c l-1^{\text {H}}$. We assume that the Z-type sclerotium represents the wild-type 

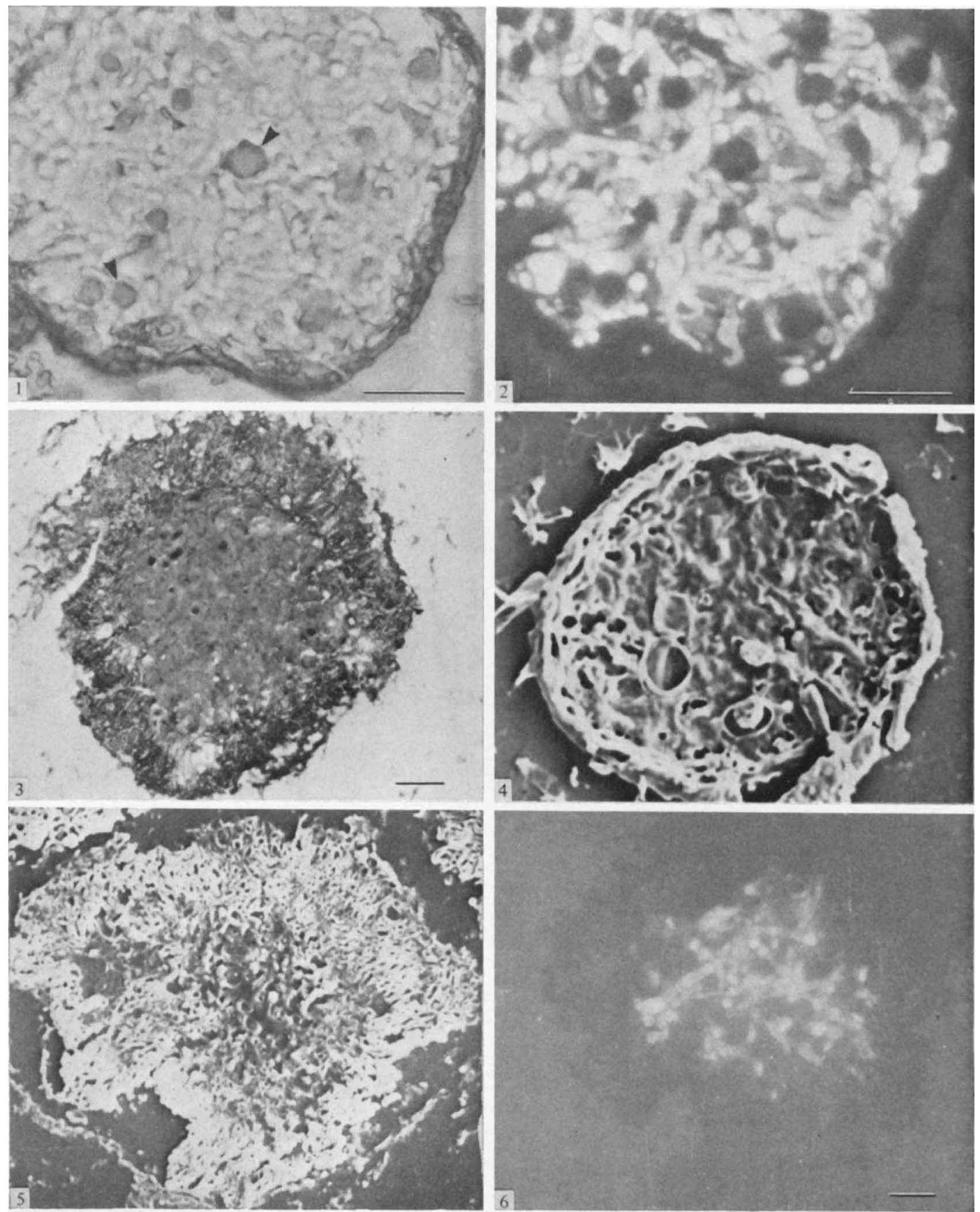

Fig. 1. Section through a Z-type sclerotium stained with periodic acid-Schiff reagent (PAS) to localize glycogen. The rind is a single layer of cells. Some large PAS-staining cells are marked (arrows). Bar marker represents $20 \mu \mathrm{m}$.

Fig. 2. The same section as in Fig. 1 treated with Photine LV and viewed under ultraviolet illumination; this reveals the hyphal structure of the medulla. Bar marker represents $20 \mu \mathrm{m}$.

Fig. 3. Section of an H-type sclerotium stained with the periodic acid-Schiff reagent (PAS). The rind is many cells thick: contrast with the similarly prepared Z-type structure in Fig. 1. However, like the specimen in Fig. 1, this section also shows large PAS-staining cells scattered in the compact, amorphous medulla. Bar marker represents $20 \mu \mathrm{m}$. 


\section{Table 1. Types of sclerotia produced by different dikaryons of Coprinus cinereus}

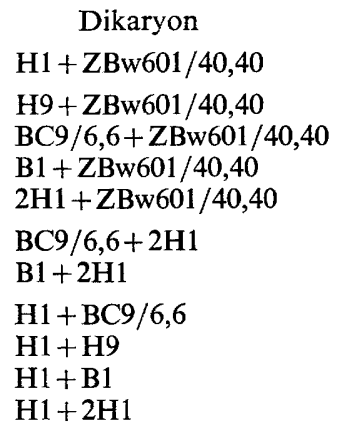

Sclerotium phenotype and
genotype of the monokaryotic
components of the dikaryon
H-type $\left(s c l-1^{\mathrm{H}}\right)+\mathrm{Z}$-type $\left(s c l-1^{+}\right)$
negative $\left(s c l-1^{\mathrm{O}}\right)+\mathrm{Z}$-type $\left(s c l-1^{+}\right)$
negative $\left(s c l-1^{\mathrm{O}}\right)+\mathrm{Z}$-type $\left(s c l-1^{+}\right)$
negative $(s c l-2)+\mathrm{Z}$-type $\left(s c l-2^{+}\right)$
negative $(s c l-3)+\mathrm{Z}$-type $\left(s c l-3^{+}\right)$
negative $\left(s c l-1^{\mathrm{O}}\right)+$ negative $(s c l-3)$
negative $(s c l-2)+$ negative $(s c l-3)$
H-type $\left(s c l-1^{\mathrm{H}}\right)+$ negative $\left(s c l-1^{\mathrm{O}}\right)$
H-type $\left(s c l-1^{\mathrm{H}}\right)+$ negative $\left(s c l-1^{\mathrm{O}}\right)$
H-type $\left(s c l-1^{\mathrm{H}}\right)+$ negative $(s c l-2)$
H-type $\left(s c l-1^{\mathrm{H}}\right)+$ negative $(s c l-3)$

Type of sclerotium produced by the dikaryon Z Z Z Z $\mathrm{Z}$ Z Z $\mathrm{H}$ $\mathrm{H}$ Z

form and is to be observed in the presence of the wild-type allele, $s c l-1^{+}$. The $s c l-1^{\mathrm{H}}$ allele is recessive to wild-type but is dominant to its 'silent' allele (which we now designate $s c l-1^{\circ}$ ) which is carried by strains $\mathrm{H} 9$ and $\mathrm{BC} 9 / 6,6$ and which gives rise to the sclerotium-negative phenotype.

This interpretation is consistent with all of the data available, but any attempt to explain the genetic basis of these variants must allow for the limitations of the genetic information so far obtained. Although $\mathrm{scl}-1^{\circ}$ exhibits the segregation expected of a single chromosomal gene (Waters et al., 1975b), the data obtained are not sufficiently extensive to allow any conclusions to be drawn about the size or complexity of the genetic entity involved. At the level of gene function it may be assumed that scl-1 represents a gene responsible for some factor involved in sclerotium morphogenesis, and that a change in the structure or production of this factor can lead either to complete blockage of sclerotium production (the $\mathrm{scl}-1^{\circ}$ phenotype) or to formation of sclerotia which are 'over-endowed' with their component tissues (the $s c l-l^{\mathrm{H}}$ phenotype). The scl-1 gene product may thus be concerned with determining the extent and type of growth made during maturation of sclerotium initials.

The origin of the sclerotial variants found in the strains described presents an interesting problem. In the present work, strains $\mathrm{H} 1$ and $\mathrm{H} 9$ were shown to carry different alleles of the scl-1 gene, while Volz \& Niederpruem (1970) demonstrated that the monokaryon H6 produced Z-type sclerotia. However, all three strains were isolated as progeny of monosporous origin from a single carpophore by P. R. Day in 1957 (Day \& Anderson, 1961). Although mutation in the scl-1 gene could have occurred during the subsequent years of vegetative culture, it seems far more likely that the varient alleles existed at the time the monokaryons were first isolated. Thus three different alleles of the scl- 1 gene were recovered from the same carpophore: $s c l-1^{+}$in $\mathrm{H}$, $s c l-1^{\mathrm{H}}$ in $\mathrm{H} 1$ and $s c l-1^{\circ}$ in $\mathrm{H} 9$. This implies that the dikaryon which formed the carpophore was a heterokaryon containing nuclei of at least three genotypes, all of which contributed to the construction of the carpophore and were represented in the population of diploid nuclei which eventually formed within the basidia. This explanation would not be appropriate if Coprinus carpophores arise from single cells of the

Fig. 4. Scanning electron micrograph of a section through a Z-type sclerotium. The rind is a single layer of cells but, in contrast to Fig. 2, the medulla appears to be amorphous. Magnification $\times 500$.

Fig. 5. Scanning electron micrograph of a section through an H-type sclerotium. The rind is many cells thick and the medulla has an amorphous appearance. Contrast with Fig. 6 where the medulla seems hyphal. Magnification $\times 320$.

Fig. 6. The same section as in Fig. 5, but here treated with aniline blue and viewed by ultraviolet fluorescence prior to its use for scanning electron microscopy. The medulla appears to contain hyphal cells. Bar marker represents $20 \mu \mathrm{m}$. 
dikaryon as suggested by Brefeld (1877). However, hyphal interactions and fusions occur extensively during early development of the carpophore primordium (Matthews \& Niederpruem, 1972; van der Valk \& Marchant, 1978), so there must be ample scope for inclusion of many different nuclei in the developing carpophore and hence a genetically chimeric structure could be formed. This would mean that the hymenium of the carpophore from which the $\mathrm{H}$-series of monokaryons were isolated initially contained at least three different types of haploid nuclei, carrying the $s c l-1^{+}, s c l-1^{\mathrm{H}}$ and $s c l-1^{\circ}$ alleles.

We thank Miss M. A. Watson and Miss E. A. Bladon for assistance; we also thank Professor W. D. P. Stewart, F.R.S., and Dr M. Crisp for the use of facilities at the Department of Biological Sciences in the University of Dundee, and Dr J. F. Peberdy for supplying the fluorescent brighteners Photine LV and Tinopal BOPT.

\section{REFERENCES}

BREFELD, O. (1877). Botanische Untersuchungen uber Schimmelpilze, vol. III. Leipzig: Felix.

Colotelo, N. (1974). A scanning electron microscope study of developing sclerotia of Sclerotinia sclerotiorum. Canadian Journal of Botany 52, 11271130.

DAy, P. R. \& Anderson, G. E. (1961). Two linkage groups in Coprinus lagopus. Genetical Research 2, 414-423.

JENSEN, W. A. (1962). Botanical Histochemistry. Principles and Practice. San Francisco: Freeman.

KEMP, R. F. O. (1975). Breeding biology of Coprinus species in the section Lanatuli. Transactions of the British Mycological Society 65, 375-388.

Lu, B. C. (1974). Meiosis in Coprinus. V. The role of light on basidiocarp initiation, mitosis and hymenium differentiation in Coprinus lagopus. Canadian Journal of Botany 52, 299-305.

Matthews, T. R. \& Niederpruem, D. J. (1972). Differentiation in Coprinus lagopus. I. Control of fruiting and cytology of initial events. Archiv für Mikrobiologie 87, 257-268.

MOORE, D. \& JIRJIS, R. I. (1976). Regulation of sclerotium production by primary metabolites in Coprinus cinereus ( $=C$. lagopus sensu Lewis). Transactions of the British Mycological Society 66, 377-382

Morimoto, N. \& ODA, Y. (1973). Effects of light on fruit-body formation in a basidiomycete, Coprinus macrorhizus. Plant and Cell Physiology 14, 217225.

Pinto-Lopes, J. \& Almeida, M. G. (1970). 'Coprinus lagopus' a confusing name as applied to several species. Portugaliae acta biologica 11, 167204.

Stewart, G. R. \& Moore, D. (1974). The activities of glutamate dehydrogenases during mycelial growth and sporophore development in Coprinus lagopus (sensu Lewis). Journal of General Microbiology 83, 73-81.

VALK, P. VAN DER \& Marchant, R. (1978). Hyphal ultrastructure in fruit-body primordia of the basidiomycetes Schizophyllum commune and Coprinus cinereus. Protoplasma 95, 57-72.

Volz, P. A. \& NiederprueM, D. J. (1970). The sclerotia of Coprinus lagopus. Archiv für Mikrobiologie 70, 369-377.

WAters, H., Butler, R. D. \& MOORE, D. (1972). Thick-walled sclerotial medullary cells in Coprinus lagopus. Transactions of the British Mycological Society 59, 167-169.

Waters, H., Butler, R. D. \& Moore, D. (1975a). Structure of aerial and submerged sclerotia of Coprinus lagopus. New Phytologist 74, 199-205.

Waters, H., MoOre, D. \& Butler, R. D. (1975b). Morphogenesis of aerial sclerotia of Coprinus lagopus. New Phytologist 74, 207-213. 